\title{
EXPANSIÓN ORTOPÉDICA DEL MAXILAR CON LA UTILIZACIÓN DE ANCLAJE ESQUELÉTICO TEMPORAL
}

\section{ORTHOPEDIC EXPANSION OF THE MAXILAR WITH THE UTILIZATION OF TEMPORAL ESQUELETIC ANCHORAGE.}

\author{
Gutierrez-Galloso Giovanna. ${ }^{1}$ * Gómez-Miranda Christian ${ }^{2}$ Obando-Romero Alonzo ${ }^{2}$ \\ ${ }^{1}$ Residente de la Especialidad de Ortodoncia UAC,PERÚ. ${ }^{2}$ Cirujano Dentista, Esp. Ortodoncia y Ortopedia de los \\ Maxilares, Docente de la Especialidad Ortodoncia UAC,PERÚ. \\ * unidents-cusco@hotmail.com
}

\begin{abstract}
Resumen
OBJETIVO. La corrección transversal del maxilar con el diagnóstico de un paladar atrésico es corregida por la expansión rápida maxilar (RME) mostrando ser un tratamiento eficaz y la opción que ha sido ampliamente utilizada para eliminar la deficiencia del maxilar. Reportamos una paciente de 10 años de edad, género femenino, estudiante sin antecedentes médicos de importancia que consulta a la facultad de odontología de la Universidad Andina del Cusco Perú. Al cuestionamiento del motivo de consulta responde "tengo los dientes muy desordenados", en el momento del examen no presenta ninguna enfermedad que contraindicara un tratamiento ortopédico. A la evaluación el paciente presenta perfil recto, hipo divergente con maloclusion clase I por problemas de espacio, con apiñamiento moderado anterior tanto superior como inferior. Al examen estomatológico sus tejidos blandos presentaban parámetros de normalidad. El paciente fue diagnosticado con maloclusión clase I con apiñamiento moderado en ambas arcadas sin presentar problemas transversales demasiado evidentes. El plan de tratamiento: Se decidió hacer la disyunción maxilar con anclaje esquelético, considerando la edad del paciente se hacen evaluaciones clínicas periódicas para realizar el monitoreo del crecimiento. Los disyuntores con anclaje esqueletal tienen menor efecto colateral. El control radiográfico debe ser continuo para corroborar que la disyunción está siendo realizada correctamente.
\end{abstract}

Palabras clave: maxilar , ortodoncia , aparatos activadores.

\begin{abstract}
AIM. The transversal correction of the maxilla with the diagnosis of an atresic palate is corrected by the rapid maxillary expansion (RME) showing to be an effective treatment and the option that has been widely used to eliminate the maxillary deficiency. We report a 10year-old female patient, a student with no medical history of importance consulting the dentistry faculty of the University Andina of Cusco. To the questioning of the reason for consultation answers "I have very disordered teeth", at the time of the examination does not present any disease that contraindicates an orthopedic treatment. On evaluation, the patient presents a straight profile, divergent hiccups with class I malocclusion due to space problems, with both upper and lower anterior moderate crowding. At the stomatological examination, his soft tissues showed normal parameters. The patient was diagnosed with class I malocclusion with moderate crowding in both arches without presenting transverse problems too evident. The treatment plan: It was decided to make the maxillary disjunction with skeletal anchorage, considering the patient's age, periodic clinical evaluations are made to perform the growth monitoring. It was concluded that although the dentosoportados and dentomucosupported circuit breakers have good clinical results, in the case that there is no support for the anchorage of the same would have as well supported options as is the case of circuit breakers with skeletal anchorage With less side effect. The radiographic control must be continuous to corroborate that the disjunction is being performed correctly.
\end{abstract}

Key words: maxilla, orthodontics, activating devices..

\section{INTRODUCCIÓN}

El término de anclaje esquelético temporal (TADS) se proporciona gracias a Mah y Bergstrand a partir de una publicación en los Estados Unidos con la finalidad de estandarizar la terminología anteriormente muy variada. ${ }^{1}$
Los TADS muestran una versatilidad de ayuda en los tratamientos ortodoncicos de propiedades menos invasivos y de relativa fácil manipulación clínica, que abarcan desde movimientos de intrusión, extrusión, verticalización y anclaje óseo para la tracción de componentes esqueletales. ${ }^{2,3}$ 
La instalación y la elección clínica de la ubicación del microtornillo deberá ser óptima para poder conseguir la estabilización y el anclaje correspondiente para realizar una correcta separación de los componentes óseos que integran el paladar duro, y cuyas características como el diámetro y el diseño del hilo miniimplante tienen un impacto distintivo sobre la estabilidad primaria, sin perder de vista la región de inserción y la calidad del hueso local, la elección del diseño y tamaño del miniimplante todas ellas cruciales para establecer una estabilidad primaria suficiente. ${ }^{4} \mathrm{La}$ corrección transversal del maxilar con el diagnostico de un paladar atresico es corregida por la expansión rápida maxilar (RME) mostrando ser un tratamiento eficaz y la opción que ha sido ampliamente utilizada para eliminar la deficiencia del maxilar. ${ }^{5} \mathrm{El}$ autor que mayores publicaciones y que más aportes tiene sobre el tema será sin lugar a dudas Andrew Hass. ${ }^{6}$ Este procedimiento además de corregir la mordida cruzada posterior, mejora los casos con problemas de perímetro de arco y corredores bucales, siendo además una alternativa en algunos casos en los que ganar espacio es posible para evitar exodoncias en el plan de tratamiento y muestra progresos repercutiendo además en la estética sonrisa. $^{7}$

Dentro de las estrategias resolutivas para las mordidas cruzadas por paladares incompetentes en sentido transversal que se mencionan en la literatura se ponen en consideración dos aspectos fundamentales de la arquitectura de la bóveda del paladar: primero, se debe evaluar el estado de maduración ósea de la sutura: línea de sutura recta, línea de sutura festoneada, línea de suturas paralela, fusión completa del hueso palatino con poca evidencia de sutura, fusión anterior de la maxila y el segundo aspecto a valorar además es la densidad ósea, ${ }^{8}$ tenemos entonces: la disyunción maxilar con anclaje dentosoportado, la disyunción maxilar con anclaje dentomucosoportado y la disyunción maxilar con anclaje esquelético. Previa a la aplicación clínica de este tipo de anclaje, se realizaron estudios previos. Se realizó un estudio de DM con anclaje esquelético en un cráneo seco, que se apoyaba en los primeros molares superiores y en dos minimplantes (MI) colocados a nivel de los premolares, obteniendo un resultado eficiente que podría evitar los efectos adversos. ${ }^{9}$ En otro estudio experimental se concluyó que el uso de dos implantes paramediales a la sutura ofrece mayor resistencia a la deformación respecto al uso de 1 onplant, con un mayor riesgo de dañar el hueso dado que su capacidad de resistencia a la tracción es limitada. Para colocar implantes a nivel de la sutura, el crecimiento debe haber finalizado, para que el hueso receptor tenga un grado de madurez y rigidez adecuada. Si se desea colocar antes de este momento, se puede colocar 1 onplant de $5 \mathrm{~mm}$ o 2 onplants de un diámetro de $3 \mathrm{~mm}$ paramediales a la sutura. ${ }^{10}$

\section{REPORTE DE CASO}

Paciente de 10 años de edad, género femenino, estudiante sin antecedentes médicos de importancia que consulta a la facultad de odontología de la Universidad Andina del Cusco. Al cuestionamiento del motivo de consulta responde "tengo los dientes muy desordenados", en el momento del examen no presenta ninguna enfermedad que contraindicara un tratamiento ortopédico. $\mathrm{Al}$ examen físico presenta crecimiento y desarrollo normal con el biotipo mesofacial.

$\mathrm{Al}$ examen facial presenta proporción de tercios, presenta simetría facial, línea media dentaria superior no coincide con la línea media facial desviada $3 \mathrm{~mm}$ a la derecha, línea media inferior, coincide con línea media facial, longitud de labio superior normal, ausencia de exposición de encías a la sonrisa.(Fig 1,2,3)

$\mathrm{Al}$ análisis funcional lo se presenta alteraciones en la respiración, deglución ni en la movilidad mandibular, presenta dentición mixta con arco superior de tipo ovoide y apiñados con discrepancia negativa de $-7 \mathrm{~mm}$ lado derecho y $-3 \mathrm{~mm}$ lado izquierdo con RMD de clase I y RMI de clase I, no registra relaciones caninas.

En el análisis radiográfico se observo una base craneal corta, patrón I esquelético, proinclinacion de incisivos superior e inferior, AFAI disminuido hipo divergente, perfil recto, mesofacial.

A la evaluación el paciente presenta perfil recto, hipo divergente con maloclusion clase I por problemas de espacio, con apiñamiento moderado anterior tanto superior como inferior. Al examen estomatológico sus tejidos blandos presentaban parámetros de normalidad. El paciente fue diagnosticado con maloclusión clase I con apiñamiento moderado en ambas arcadas.

El pronóstico del paciente se considera favorable para hacer la expandió rápida palatina con aparato ortopédico.

Los objetivos del tratamiento son corregir la atresia maxilar, y por consiguiente el apiñamiento dentario, establecer un perfil armónico, disminuir la discrepancia esquelética maxilomandibular.

En el plan de tratamiento se decidió hacer la disyunción maxilar con anclaje esquelético, considerando la edad del paciente se hacen evaluaciones clínicas periódicas para realizar el monitoreo del crecimiento (Fig4).

\subsection{PROGRESO DEL TRATAMIENTO:}

Se realizó la instalación de los micro implantes de $6 \mathrm{~mm}$ a cada lado del rafe medio palatino seguido de la toma de impresión para la confección de una placa tipo has para realizar la disyunción, una vez instalado en boca se realizó a los primeras activaciones de 4 cuartos de vuelta por día durante los 7 días posteriores a la instalación, evidenciándose 
el diastema al 4to día de activada la placa y evidenciándose la ruptura palatina al examen radiográfico. (Fig5)

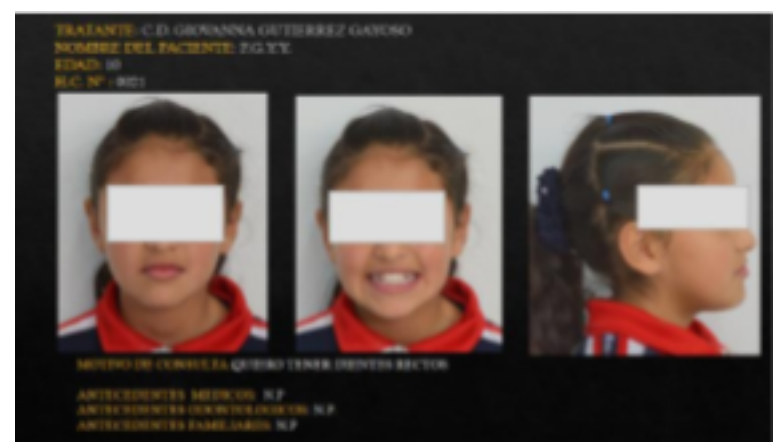

Fig. 1. FOTOGRAFÍAS EXTRAORALES

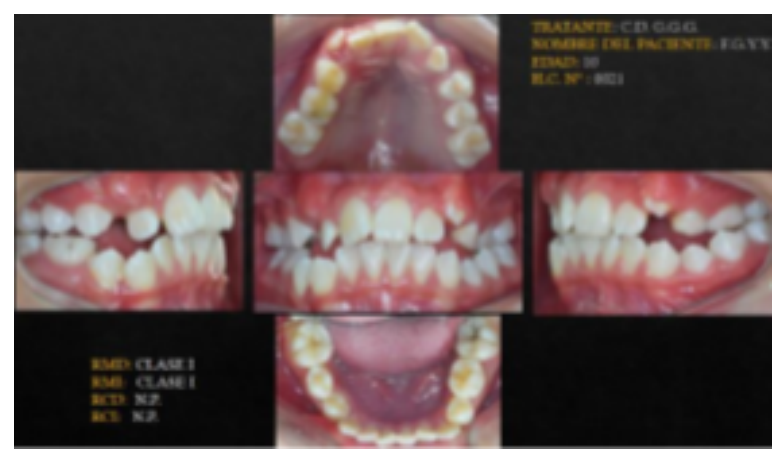

Fig. 2. FOTOGRAFÍAS INTRORALES

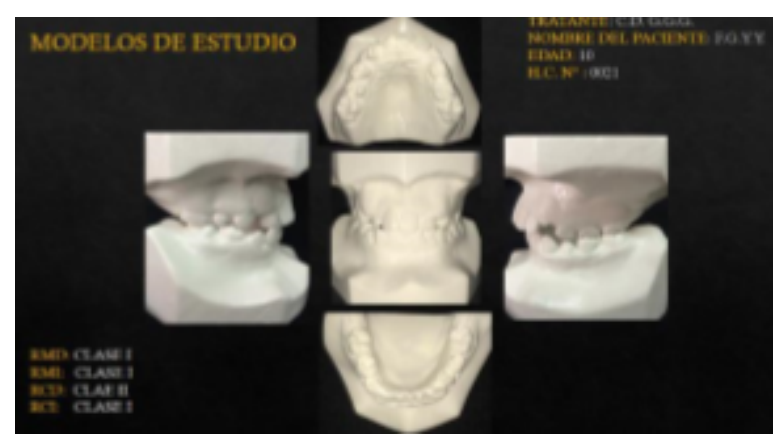

Fig. 3. FOTOGRAFÍAS DE MODELOS DE ESTUDIO

\section{DISCUSIÓN}

Con el uso de anclajes dentosoportados como en el caso del empleo de los aparatos tipo Haas, además de los cambios transversales deseables, la expansión ortopédica (EO) del maxilar produce cambios en los planos sagital y vertical. ${ }^{11}$ Durante la expansión ortopédica, la carga liberada provoca elevada compresión de ligamento periodontal con inclinación

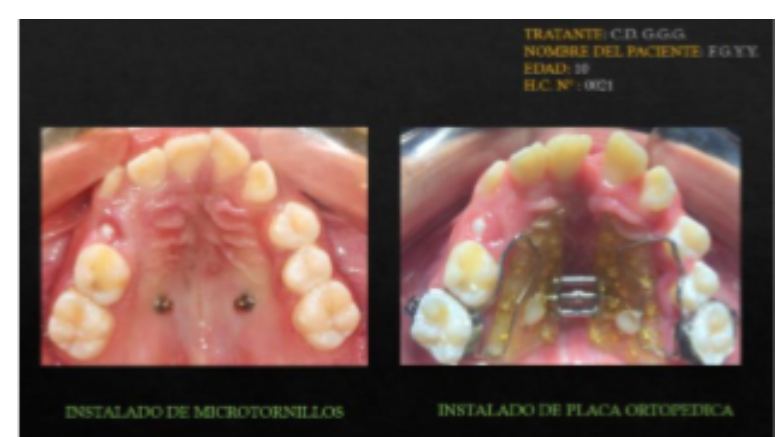

Fig. 4. INSTALADO DE MICROIMPLANTES Y DISYUNCIÓN A LOS 7 DÍAS

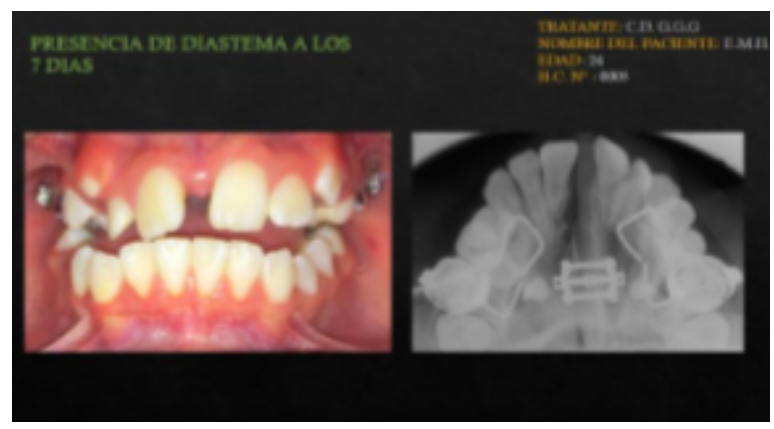

Fig. 5. CONTROL RADIOGRÁFICO

lateral de los dientes postero superiores, de este modo, la EO corresponde a expansión esquelética y como efecto colateral, también, ortodóncica, ${ }^{12,13}$ que en algunos casos es indeseable o poco deseable porque a veces hay poco material dental donde se podría anclar el aparato como es en el caso clínico reportado. Se ha reportado además otros efectos adversos como aumento de inclinación osea alveolar, ${ }^{14-16}$ aumento de periodos de contención post expansión16, reducción de la placa osea y el nivel de cresta osea de los dientes de anclaje ${ }^{17}$ y reabsorción radicular de los mismos. ${ }^{18}$ Otro efecto de los aparatos dentosortados con férula acrílica como es el tipo Mcnamara, se entiende que la cubierta acrílica oclusal que describe su diseño biomecánico ayuda a fijar el material dental presente en la arcada, ayudando a la expansión, evitando las interferencias oclusales durante la EO, proporcionando una expansión simétrica pero inhibe el desenvolvimiento vertical de los dientes posteriores, indicándose para pacientes con crecimiento vertical. ${ }^{19}$ Haas planteó que si se añade una cubierta de acrílico palatina para apoyar el aparato, produciría mayor movimiento de traslación y por lo tanto menor inclinación dentaria; con esto las fuerzas se dirigen contra los dientes, tejido blando y duro del paladar, ${ }^{20}$ después de la tercera vuelta completa del tornillo, los dientes reciben el impacto de la EO, caracterizándose desde entonces, en una relación directa entre el diastema abierto interincisivo y la cantidad de efecto ortopédico inducido por la expansión, lo 
que éste disyuntor puede liberar fuerzas de $10000 \mathrm{~g},{ }^{21}$ cifra 10 impensable en pacientes que tienen poco material dental en boca o en situaciones clínicas de recambio dental fisiológico en etapas intertransicionales. Hablando de resultados clínicos el aparato Haas podría causar mayor grado de inflexión vestibular de los dientes de anclaje $\left(3,5^{\circ}\right.$ para la primera molar) vs el Hyrax $\left(1.6^{\circ}\right)$, aunque clínicamente no es relevante. ${ }^{22}$ Comprobado está que el anclaje esquelético ofrece un mejor control vertical y sagital. ${ }^{23}$ En cuanto al protocolo de activación de los aparatos con anclaje esqueletal se ha comentado que oscila entre una a cuatro activaciones por día, ${ }^{24}$ se ha encontrado además que activaciones por encima de las 4 activaciones por días podrían producir microfracturas oseas y conducir a la perdida de los implantes. ${ }^{24}$

\section{CONCLUSIÓN}

Los disyuntores con anclaje esqueletal con menor efecto colateral. El control radiográfico debe ser continuo para corroborar que la disyunción está siendo realizada correctamente.

\section{Referencias}

1 Korrodi Ritto A. Skeletal anchorange with microimplants. Facies, Centro de Estudios Armonía Facial, Lda. Portugal. Mayo 2007

2 H. Wehrbein and P. Göllner, "Miniscrews or palatal implants for skeletal anchorage in the maxilla: comparative aspects for decision making," World Journal of Orthodontics, vol. 9, no. 1, pp. 63-73, 2008.

3 T. C. K. Lee, M. T. C. Leung, R. W. K. Wong, and A. B. M. Rabie, "Versatility of skeletal anchorage in orthodontics," World Journal of Orthodontics, vol. 9, no. 3, pp. 221-232, 2008.

4 Wilmes B, Ottenstreuer S, Su YY, Drescher D. Impact of implant design on primary stability of orthodontic miniimplants. J Orofac Orthop. 2008 Jan;69(1):42-50

5 Puebla R., Lorenzo. Manejo de la dimensión transversal (expansión) por medio de microtornillos (TADS). Revista mexicana de ortodoncia. Vol. 3, Núm. 1 Enero-Marzo 2015 pp 33-38

6 Haas AJ. The treatment of maxillary defi ciency by opening the midpalatal suture. Angle Orthod. 1965; 35: 200217.

7 Guls,ilay Sayar Torun. Soft tissue changes in the orofacial region after rapid maxillary expansion A cone beam computed tomography study. J Orofac Orthop. 2016 Dec12.

8 Siqueira D:F., Almeida R., Henriques J. F. C. Estudio comparativo por meio de análisecefalométricaem norma frontal, dos efeitosdentoesqueléticos produzidos por tres tipos de expansores palatinos. Rev. Dent. PressOrtodon. Ortoped. Facial. Maringá, v.7, n.6, p. 27-47, 2002.

9 Garib D.G., Navarro R.L y cols.Expansao rápida da maxila ancorada em implantes - uma nova proposta para expansao ortopédica na dentadura permanente. Rev. Dent. Pressortodon. Ortoped. Facial, maringá, v.12, n.3, p. 75-81, 2007.
0 Gedrange T, Kobel C, Harzer W. Deformación del paladar duro en un modelo animal tras someter a carga cuasiestática para estimular los implantes como anclaje. Eur J Orthod. 23: 349-54, 2001.

1 Podesser B., Williams S. y cols.Evaluation of theeffects of rapidmaxillary expansión in growingchildrenusingcompute rtomographyscanning: a pilotstudy.EurJournal of Orthod 29 37-44. 2007

12 Rungcharassaeng K., CarusJ.M., Kan J.Y.K., Kim J., Taylore G. Factor affectingbuccalbonechanges of maxillary posterior teethafterrapidmaxillary expansión. Am J OrthodDentofacialOrthop132: 428. e 1-428. e 8, 2007.

13 Korkhaus G. Discussion of report: a review of orthodonticresearch (19461950). International dental journal. 3: 356, 1953.

14 Kartalian A, Gohl E, Adamian M, Enciso R. Cone-beam computerized tomography evaluation of the maxillary dentoskeletal complex after rapid palatal expansion. Am J Orthod Dentofacial Orthop 2010;138:486-92

15 Lione R, Franchi L, Cozza P. Does rapid maxillary expansion induce adverse effects in growing subjects? Angle Orthod 2013;83:172- 82

16 Parr JA, Garetto LP, Wohlford ME, Arbuckle GR, Roberts WE. Sutural expansion using rigidly integrated endosseous implants: an experimental study in rabbits. Angle Orthod 1997;67:283-90

17 Garib DG, Henriques JF, Janson G, de Freitas MR, Fernandes AY. Periodontal effects of rapid maxillary expansion with tooth-tissueborne and tooth-borne expanders: a computed tomography evaluation. Am J Orthod Dentofacial Orthop 2006;129:749-58.

18 Erverdi N, Okar I, Kuck ukkeles N, Arbak S. A comparison of two different rapid palatal expansion techniques from the point of root resorption. Am J Orthod Dentofacial Orthop 1994;106:47-51

19 Mc Namara Junior J.A., Brudon W.L. Bondedrapidmaxillary expansión appliances: Orthodontic and Orthopedictreatment in the mixed dentition. 2da Ed. Ann Arbor, NeedhamPress. Cap. 8 p.145-169, 1993. 5 yuli.

20 Rodriguez, E. De la Impresión a la Activación en Ortodoncia y Ortopedia: Aparatos de elección para disyunción y expansión.1ra Ed. 2011

21 CalliandraMoura Pereira, Disjuntor Palatino Tipo Haas. Dental PressOrtodonOrtop Facial. 8(2): 24-27, 2001

22 Awuapara-Flores S, Meneses-López A. Evaluación de los cambios esqueléticos verticales post- tratamiento ortodóntico de la expansión maxilar rápida con aparato de Haas y Hyrax. RevEstomatol Herediana. 19(1):12-17, 2009.

23 Garib D.G., Navarro R.L y cols.Expansao rápida da maxila ancorada em implantes - uma nova proposta para expansao ortopédica na dentadura permanente. Rev. Dent. Pressortodon. Ortoped. Facial, maringá, v.12, n.3, p. 75-81, 2007.

24 Lee K, Park Y, Park J, et al. Miniscrew-assisted nonsurgical palatal expansion before orthognathic surgery for a patient 
with severe mandibular prognathism. Am J Orthod Dentofacial Orthop 2010;137:830-9.

Recibido: 16 de Mayo de 2018.

Aceptado: 17 de Junio de 2018. 
